\title{
X-Ray or Neutron Reflectivity: Limitations in the Determination of Interfacial Profiles
}

\section{Citation}

Pershan, Peter S. 1994. X-ray or neutron reflectivity: Limitations in the determination of interfacial profiles. Physical Review E 50(3): 2369-2372.

\section{Published Version}

doi:10.1103/PhysRevE.50.2369

\section{Permanent link}

http://nrs.harvard.edu/urn-3:HUL.InstRepos:10357532

\section{Terms of Use}

This article was downloaded from Harvard University's DASH repository, and is made available under the terms and conditions applicable to Other Posted Material, as set forth at http:// nrs.harvard.edu/urn-3:HUL.InstRepos:dash.current.terms-of-use\#LAA

\section{Share Your Story}

The Harvard community has made this article openly available.

Please share how this access benefits you. Submit a story.

\section{Accessibility}




\title{
X-ray or neutron reflectivity: Limitations in the determination of interfacial profiles
}

\author{
P. S. Pershan \\ Division of Applied Sciences and the Physics Department, Harvard University, Cambridge, Massachusetts 02138
}

(Received 19 January 1994)

\begin{abstract}
Although there are an increasing number of proposals for model independent methods by which an index of refraction profile can be extracted from either $x$-ray or neutron reflectivity data, for many systems there are fundamental limitations to the uniqueness of profiles so determined. The problem arises from the fact that all methods of analysis are ultimately hampered by the fact that the phase information obtained by a reflectivity measurement is incomplete. We discuss the conditions under which uniqueness is, and is not, possible without some external (other than reflectivity) information.
\end{abstract}

PACS number(s): 61.10. - i, 61.12. $-\mathrm{q}$

The increasingly widespread use of $x$-ray and neutron reflectivity to probe liquid and solid surfaces [1-7] has stimulated various approaches for extracting the interfacial index of refraction profile, $n^{2}(z) \equiv 1-\chi(z)$, from the measured reflectivity [8-18]. The simplest and most ubiquitously used kinematic or Born approximation $\left(\alpha^{\prime}=\alpha\right)$, is illustrated in Fig. 1 for either $x$ rays or neutrons of wavelength $\lambda$, incident from vacuum on a planar surface of a material whose bulk scattering density is $\chi(-\infty)$. For a plane wave incident at a grazing angle $\alpha$, the ideal Fresnel reflectivity from an abrupt flat interface is designated as $R_{F}(Q)$, where $Q=(4 \pi / \lambda) \sin \alpha$. When $\alpha$ is large enough that the normal component of the refracted wave vector in the material is essentially equal to the normal component of the incident wave vector, i.e., $k_{z}^{\prime}$ $=\sqrt{k_{z}^{2}-(2 \pi / \lambda)^{2} \chi(-\infty)} \cong k_{z}$, the reflectivity from an interface with some general profile can be approximated by [19]

$$
\begin{aligned}
R(Q) & \cong R_{F}(Q)|\Phi(Q)|^{2} \\
& =R_{F}(Q)\left|\frac{1}{\chi(-\infty)} \int d z \frac{d\langle\chi(z)\rangle}{d z} e^{i Q z}\right|^{2},
\end{aligned}
$$

where $\Phi(Q)$ is the complex structure factor of the surface. Although we will not go into the details here, the quantity $\langle\chi(z)\rangle$ is the average of $\chi(x, y, z)$ over the projection of the $x$-ray or neutron correlation area on an $x-y$ plane at some height $z$ along the surface normal $[20,21]$. When $\chi(-\infty)$ is positive, which is always the case for $\mathrm{x}$ rays, the region in which Eq. (1) is valid is typically of the order of $\alpha \geq 4 \sqrt{\chi(-\infty)}$ or $5 \sqrt{\chi(-\infty)}$, where the critical angle $\alpha_{c}=\sqrt{\chi(-\infty)}$. A general goal of all publications dealing with reflectivity has been to develop a systematic procedure by which $\langle\chi(z)\rangle$, or $d\langle\chi(z)\rangle / d z$, can be extracted from the measured $R(Q)$. The principal obstacle, which has been the problem since the dawn of $x$-ray diffraction, is that, for surface profiles in which $R(Q)$ is well represented by the kinematic approximation, $R(Q)$ is proportional to the absolute value of the Fourier transform of the density profile and is insensitive to the phase information in $\Phi(Q)$ that is necessary to the inversion process for extracting $\langle\chi(z)\rangle[22]$.

In the absence of phase information the most common stratagem has been to represent a physically motivated model profile $\left\langle\chi^{\operatorname{model}}(z)\right\rangle$ by some parametrized function. The absolute value of the difference between the reflectivity calculated from this model, $R^{\text {model }}(Q)$, and the measured reflectivity, the so called $\chi^{2}$, is then minimized by one or another fitting routine. So long as the most important physical information is from the region with $Q^{2} \gg(4 \pi / \lambda)^{2}|\chi(-\infty)|, R^{\text {model }}(Q)$ can be calculated using Eq. (1); however, if the small $Q$ region is important other techniques such as the matrix formulation of Parratt should be used [23]. Unfortunately, using this type of fitting technique one never knows whether the solution found is the right one, or whether some other "guess," or some other parametrization of a different function, might yield a different, and possibly superior, $\langle\chi(z)\rangle$. In particular, all fitting procedures are notorious for sometimes converging around some "local minimum" in $\chi^{2}$. Faced with these uncertainties, different groups have tried to develop alternative, model independent procedures that, hopefully, would be more definitive [8-18]. By way of example consider the recent work by Zhou and coworkers $[14,15]$ in which they point out that in the small $Q$ region, where Eq. (1) is not accurate, $R(Q)$ is sensitive to the phase of $\Phi(Q)$ and in principle this allows a unique determination of $\langle\chi(z)\rangle$; however, there are practical limitations to when this will work. The purpose of this note is to be more explicit about limitations on the uniqueness of profiles extracted by their procedure, as well as all other model independent and model dependent fitting procedures.

Returning to the kinematic approximation, Eq. (1), it is obvious that when $\langle\chi(z)\rangle$ is a real function, which is true when the $x$-ray (neutron) energy is not near to an atomic (nuclear) resonance, one can always reflect $d\langle\chi(z)\rangle / d z$ about an arbitrary plane $z=z_{0}$ such that

$$
\begin{aligned}
& \left|\frac{1}{\chi(-\infty)} \int d z \frac{d\langle\chi(z)\rangle}{d z} e^{i Q z}\right| \\
& =\left|\frac{-1}{\chi(-\infty)} \int d z \frac{d\left\langle\chi\left(z_{0}-z\right)\right\rangle}{d z} e^{i Q z}\right| .
\end{aligned}
$$

Consequently, so long as one is strictly limited to the kinematic approximation there is no way by which any analytical technique can distinguish between a profile $\langle\chi(z)\rangle$ and a "reflected" profile $\left\langle\chi^{R}(z)\right\rangle$ that is related to $\langle\chi(z)\rangle$ by 


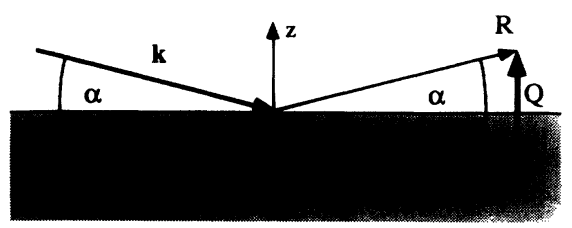

FIG. 1. Kinematics of the reflectivity geometry discussed in the text.

$$
\left[d\left\langle\chi^{R}(z)\right\rangle\right] / d z=-\left[d\left\langle\chi\left(z_{0}-z\right)\right\rangle\right] / d z .
$$

An example of profiles $\langle\chi(z)\rangle$ and $\left\langle\chi^{R}(z)\right\rangle$ for which Eq. (1) yields identical values of $R(Q)$ is shown in Fig. 2.

In practice these two profiles can be distinguished by a modified version of the kinematic approximation in which refraction effects are taken into account by replacing $Q=2 k_{2}$ in the phase factor by $Q^{\prime}$ $=2 \sqrt{k_{z}^{2}-(2 \pi / \lambda)^{2} \chi_{n}}, n=1,2$, where $\chi_{2} \equiv \chi(-\infty)$ and $\chi_{1}=\eta \chi(-\infty)$. Since the value of $Q^{\prime}$ within the surface layer $L$ is different for these two profiles, the phase governing the interference between the reflections from the two surfaces is also different for the two profiles. This is the essence of the Zhou et al. argument [14,15] that small angle data contains phase information facilitating discrimination between these two profiles. It is illustrated in Fig. 3(a) where the ratio $R(Q) / R_{F}(Q)$ has been calculated using the exact Parratt formulation for profiles like those shown in Fig. 2. For this example the value of $\langle\chi(-\infty)\rangle$ is that of $\mathrm{H}_{2} \mathrm{O}$ for $8-\mathrm{keV} \mathrm{x}$ rays [i.e., $\left.(4 \pi / \lambda) \alpha_{c_{0}}=0.0217 \AA^{-1}\right]$, and the other parameters are $L=500 \AA$ and $\eta=0.1$. Although the two reflectivities approach one another for $Q \geq 0.1 \AA^{-1}$, as pointed out by Zhou et al. the differences are unambiguous for smaller $Q$. Unfortunately, as illustrated by the same calculation for $L=100 \AA$ [Fig. 3(b)] and $30 \AA$ [Fig. 3(c)], although the difference between the reflectivities from the two profiles can easily be measured when $L$ is sufficiently large, it becomes vanishingly small for smaller $L$. For

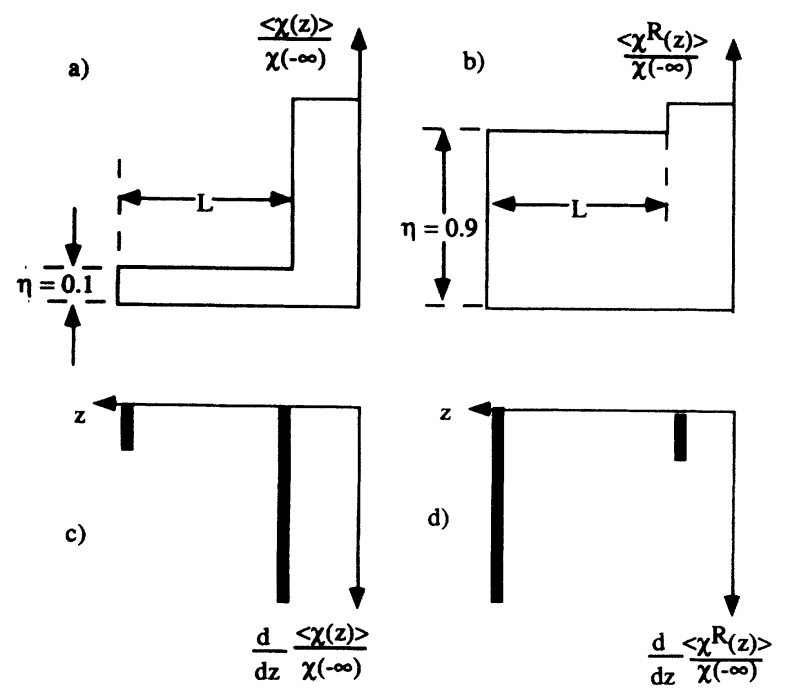

FIG. 2. Illustration of profiles $\langle\chi(z)\rangle$ (a) and $\left\langle\chi(z)^{R}\right\rangle$ (b), where there is a value of $z_{0}$ (not shown) for which $d\left\langle\chi^{R}(z)\right\rangle / d z$ (d) is equal to $d\left\langle\chi\left(z_{0}-z\right)\right\rangle / d z$ (c). Using the kinematic approximation the $R(Q)$ calculated from these two profiles is identical.
$L=100 \AA$ the feasibility of measuring differences between the two profiles depends delicately on the quantitative accuracy of the measurement, since even though counting statistics might be sufficient, systematic measurement errors near the critical angle will often become the limiting consideration. For $L=30 \AA$ the distinction between the $R(Q)$ from the two profiles has become unmeasurably small.

The point to be emphasized is that if $L^{-1} \gg \lambda^{-1} \sqrt{|\chi(-\infty)|}$ the phase information necessary for distinguishing between profiles of these two types only appears in $\Phi(Q)$ for the large $Q$ region where the kinematic approximation is valid. Note that $\Phi(Q)$ is defined as an integral over the real electron density profile and is not dependent on the kinematic approximation described by Eq. (1). Since the phase information is lost in the kinematic approximation, it follows that there is no possibility for developing a model independent method of analysis capable of extracting a unique $\langle\chi(z)\rangle$ from $R(Q)$ when the profiles are asymmetric with length scales less than $\sim \lambda(|\chi(-\infty)|)^{-1 / 2}$. This can be made slightly more quantitative by expanding $\exp [i Q z]$ in powers of $\left(z-z_{0}\right)$ :

$$
\begin{aligned}
\Phi(Q) & =[1 / \chi(-\infty)] \int d z[d\langle\chi(z)\rangle / d z] e^{i Q z} \\
& =\left[1+\sum_{n=2}^{\infty} \frac{i^{n} Q^{n}}{n !} \Xi_{n}\right] e^{i Q z_{0}} \\
& \cong\left\{\left[1-\frac{Q^{2} \Xi_{2}}{2}+\cdots\right]-i\left[\frac{Q^{3} \Xi_{3}}{6}-\cdots\right]\right\} e^{i Q z_{0}},
\end{aligned}
$$

where $\Xi_{n} \equiv[1 / \chi(-\infty)] \int\left(z-z_{0}\right) n[d\langle\chi(z)\rangle / d z] d z$ and $z_{0}$ is chosen such that $\Xi_{1}=0$; by definition $\Xi_{0} \equiv 1$. A minimum necessary criterion, although not sufficient, for claiming to have determined a unique profile associated with a given $R(Q)$ is the ability to perform a measurement capable of detecting the $\Xi_{3}$ term. This statement is independent of the analytical method by which $R(Q)$ is related to $\langle\chi(z)\rangle$. As explained above, when the length scale of the interfacial structure is large compared to the

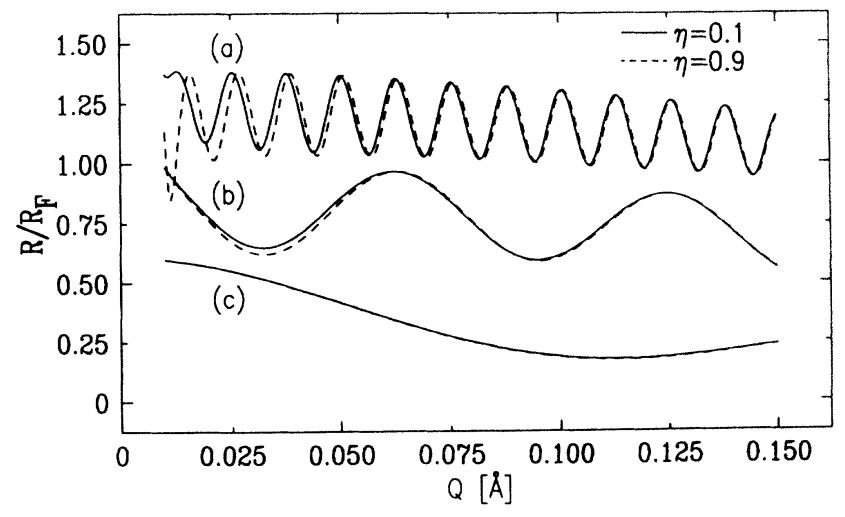

FIG. 3. $R(Q) / R_{F}(Q)$ calculated for profiles $\langle\chi(z)\rangle$ obtained by Gaussian smearing of profiles ( $\sigma=3 \AA$ ) like that of Figs. 2(a) ( and 2 (b) $(---) ; L=500 \AA$ (curve a), $100 \AA$ (curve b), and $30 \AA$ (curve c). Data for curves a and c are displaced by \pm 0.4 for clarity. 
critical angle the experimental reflectivity technique is not usually sensitive to the phase information contained in $\Phi(Q)$; an exception to this will be explained below.

The essential character of the absent phase information is illustrated even more explicitly in Fig. 4, where theoretical profiles are calculated in the kinematic approximation when the scattering amplitude for a Gaussian profile is modified by arbitrary phase variations of the form $\varphi(Q) \equiv(\Gamma Q)^{3}$. Taking $R(-Q)=R(Q)$ and $\mid R(Q) /$ $\left.R_{F}(Q)\right|^{1 / 2}=\exp \left[-(\sigma Q)^{2} / 2\right]$, Fig. 4 is a plot of

$$
\frac{-1}{\chi(-\infty)} \frac{d\langle\chi(z)\rangle}{d z}=\int_{-\infty}^{\infty}\left|\frac{R(Q)}{R_{F}(Q)}\right|^{1 / 2} e^{i(\Gamma Q)^{3}} e^{i Q_{z}} d Q
$$

for $\sigma=4 \AA$ and $\Gamma=\sigma$ (curve $a$ ), $0.75 \sigma$ (curve $b$ ), $0.5 \sigma$ (curve $c$ ), and 0 (curve $d$ ); the first minimum of the oscillations occurs for $Q \sim \pi / \Gamma$ in curves a and $b$. Note that the imaginary part of the integral is identically zero. Although one might expect that for sufficiently large $\Gamma$ some such profiles will have unphysical values of $\langle\chi(z)\rangle$, this does not seem to be the case for these examples. The unfortunate lesson to be drawn from Fig. 4 is that the effects of unknown phase variations can be quite profound and without some other physical information there are multiple possible profiles that could all yield the same reflectivity. This example illustrates that within the kinematic approximation it is not even possible to exclude the possibility that a reflectivity as simple as $R(Q) / R_{F}(Q)=\exp \left(-Q^{2} \sigma^{2}\right)$ could correspond to an oscillatory profile. Of course more exact ways of calculating $R(Q)$ from $\langle\chi(z)\rangle$, such as the Parratt method, will resolve such ambiguities when the period of the oscillations is large enough.

A slight generalization of the interfaces being discussed so far is to consider the problem where $L$ is sufficiently large that it makes sense to discuss separately the fine structure of one or the other of the individual interfaces at $z=0$ or $L$. In this case the kinematic limit can be expressed as

$$
\begin{aligned}
R(Q) / R_{F}(Q)= & \left|\Phi_{0}(Q)+\Phi_{L}(Q) e^{i Q L}\right|^{2} \\
= & \left|\Phi_{0}(Q)\right|^{2}+\left|\Phi_{L}(Q)\right|^{2}+2\left|\Phi_{0}(Q) \| \Phi_{L}(Q)\right| \\
& \times \cos \left\{Q L+\left[\varphi_{0}(Q)-\varphi_{L}(Q)\right]\right\}
\end{aligned}
$$

where $\Phi_{0, L}(Q) \equiv\left|\Phi_{0, L}(Q)\right| \exp \left(i \varphi_{0, L}(Q)\right.$ are the complex structure factors of the two interfaces. Lurio et al. applied this expression to study the fine structure of the superfluid ${ }^{4} \mathrm{He}$-vapor interface for thick films adsorbed onto an atomically flat $\mathrm{Si}(111)$ surface that was passivated with hydrogen $[24,25]$. They calculated the complex form for $\Phi_{0}(Q)$ ( $\mathrm{Si}-{ }^{4} \mathrm{He}$ interface) from an atomic model and interpreted the measured nonlinear dependence of $Q L+\left[\varphi_{0}(Q)-\varphi_{L}(Q)\right]$ in a way that essentially determined $\Xi_{3}$. This is potentially a rather powerful technique; however, it requires well defined substrates [i.e., $\Phi_{0}(Q)$ well characterized] and interfaces whose internal length scales are such that $\Xi_{3} Q^{3}$ is large enough to be measured within the accessible range of $Q$. To the best of our knowledge, Lurio's work is the only known application of the approach.

Sanyal et al. [26] proposed a variation on this tech-

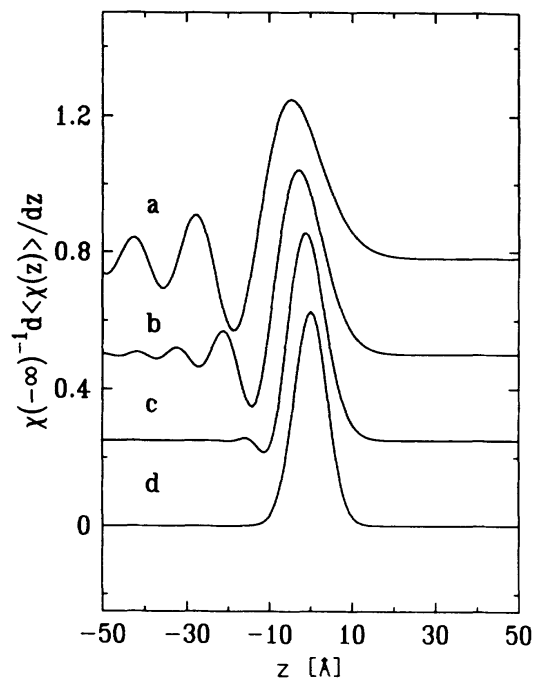

FIG. 4. Scattering profiles $[\chi(-\infty)]^{-1} d\langle\chi(z)\rangle / d z$ that are obtained by multiplying the real scattering amplitude $\left[R(Q) / R_{F}(Q)\right]^{1 / 2}$ $=\exp \left[-(Q \sigma)^{2} / 2\right]$ by phase factors of the form $\exp \left[-i(\Gamma Q)^{3}\right]$ for $\Gamma=\sigma$ (curve a), $0.75 \sigma$ (curve b), $0.5 \sigma$ (curve c) and 0 (curve d); curves b, $c$, and $\mathrm{d}$ are successively displaced by 0.25 for clarity.

nique in which they proposed using a single crystal of $\mathrm{Ge}$ as the substrate to measure the complex $\Phi_{0}(Q)$ for adsorbed films. By measuring $R(Q)$ as a function of $\lambda$, where $\lambda$ spanned the Ge $K$ absorption edge, they proposed making use of the known phase variation in the atomic scattering factor of $\mathrm{Ge}$ to calculate $\Phi_{0}(Q, \lambda)$ $=\left|\Phi_{0}(Q, \lambda)\right| \exp \left\{i\left[\varphi_{0}(Q)\right]\right\} \exp \left\{i \Delta \varphi_{\mathrm{Ge}}(Q, \lambda)\right\}[26]$. In the simplest approach the interference term in Eq. (6) would take on the form $\cos \left\{Q L+\left[\varphi_{0}(Q)-\varphi_{L}(Q)\right]\right.$ $\left.+\Delta \varphi_{\mathrm{GE}}(\lambda)\right\}$, where $\Delta \varphi_{\mathrm{Ge}}(Q, \lambda)$ is the known phase of the $\mathrm{Ge}$ atomic scattering factor. From the $\lambda$ dependence of $R(Q)$ it would then be possible to extract $\left[\varphi_{0}(Q)-\varphi_{L}(Q)\right]$, and they argued that the structure of $\varphi_{0}(Q)$ could be sufficiently well known that the form of $\varphi_{L}(Q)$ could be extracted. Unfortunately, this approach, like the one used by Lurio et al. for ${ }^{4} \mathrm{He}$, ultimately depends on knowledge of subtle features of the substrate and it is difficult to make a general statement about how widely it will be applicable without more information on how well the interface to the Ge substrate can be known.

A complication of the interference geometry which will sometimes limit the applicability of the interference technique has to do with correlations, or lack of such, between height-height variations of the two interfaces. It is well known that, if $z=h_{i}(x, y)(i=0$ or $L)$ describes the local position of the $i$ th interface, the reflectivity decreases by a Debye-Waller-like factor $\left\langle\left|\Phi_{i}(Q)\right|^{2}\right\rangle$ $\sim \exp \left(-\sigma_{i}^{2} Q^{2}\right) \quad$ where $2 \sigma_{i}^{2}=\left\langle\left[h_{i}(0,0)-h_{i}(x, y)\right]^{2}\right\rangle$. Averages by which the $\sigma_{i}$ are defined are calculated by averaging $\left\langle h_{i} h_{i}(x, y)\right\rangle$ over the projection of the $\mathrm{x}$-ray or neutron correlation volume on the $x, y$ plane. The assumption often made, but only sometimes true, is that the Debye-Waller factor governs the amplitude of the interference term $2\left\langle\Phi_{0}(Q) \Phi_{L}(Q)\right\rangle \sim \exp \left(-\left[\sigma_{0}^{2}+\sigma_{L}^{2}\right] Q^{2} /\right.$ $2)$; in fact this is a lower limit and the interference term always decays more rapidly then this [27]. When any of the correlation lengths for the $\left\langle h_{i} h_{j}(x, y)\right\rangle$ correlation 
functions is comparable to the projection of the $\mathrm{x}$-ray, or neutron, coherence area on the $x-y$ plane these DebyeWaller factors can vary with $Q$. In the simplest approximation such variations only affect the amplitude of the interference fringes, not the phase; however, if such an effect is important, and not properly included in the analysis, it will lead to systematic errors in any fitting procedure.

To summarize, we have shown that, since the kinematic approximation by which the $x$-ray or neutron reflectivity can be related to the index of refraction profile does not preserve the phase of the surface structure factor, model independent methods for extracting the profile from reflectivity data are ultimately dependent on small angle data for which refraction effects cannot be neglect$e d$. If the characteristic length scale (along the surface normal) for a surface profile is $L$, and if $L \ll \lambda /(\sqrt{|n-1|})$, the important phase information will only manifest itself in the scattering amplitude at large $Q \sim \pi / L$ where the kinematic approximation is valid and where the phase information is unobservable. Consequently, model independent methods for extracting the profile are fundamentally limited to broad interfaces.

A second subtle consequence of the absence of phase information is that, since the reflectivities due to scattering amplitudes $\left|R(Q) / R_{F}(Q)\right|^{1 / 2}$ and $\left|R(Q) / R_{F}(Q)\right|^{1 / 2}$ $\times \exp [i \varphi(Q)]$ are indistinguishable, when considered in the context of the kinematic approximation, it is necessary to consider whether oscillatory profiles, like those shown by Fig. 4, are physically reasonable. The fact that this is true even for Gaussian profiles is particularly surprising and, to the best of our knowledge, has not previously been recognized.

Although the interference geometry, in which the profile to be determined corresponds to one interface of a slab of thickness $L$ adsorbed on a substrate, does contain phase information, this technique requires detailed information both on the structure of the interface with the substrate and on correlations between the positional variations of the two interfaces.

The final point to be made is that, even though $x$-ray and neutron reflectivity do not usually contain enough information for a model independent determination of the interfacial profile, they are still very powerful techniques. When intelligent recognition of the limitations of the reflectivity technique is combined with other available information it is usually possible to extract the physically meaningful quantitative information on interfacial structures that would not be available by any other technique.

The research described here was supported by Grants No. NSF-DMR-91-13782 and No. NSF-DMR-89-20490. We would also like to acknowledge helpful discussions with Tom Russell and Jan Petersen about various aspects of this manuscript.
[1] R. A. Cowley, in Equilibrium structure and properties of surfaces and interfaces, edited by A. Gonis and G. M. Stocks (Plenum, New York, 1992), p. 1.

[2] T. J. Crowley, E. M. Lee, E. A. Simister, and R. K. Thomas, Physica B 173, 143 (1991).

[3] D. Jacquemain, S. G. Wolf, F. Leveiller, M. Deutsch, K. Kjaer, J. Als-Nielsen, M. Lahav, and L. Leiserowitz, Angew. Chem. Int. Ed. Engl. 31, 130 (1992).

[4] J. Penfold and R. K. Thomas, J. Phys. Condens. Matter 2, 1369 (1990).

[5] P. S. Pershan, Physica A 200, 50 (1993).

[6] I. K. Robinson and D. J. Tweet, Rep. Prog. Phys. 55, 599 (1992).

[7] T. P. Russell, Mater. Sci. Rep. 5, 171 (1990).

[8] D. S. Sivia, W. A. Hamilton, G. S. Smith, T. P. Rieker, and R. Pynn, J. Appl. Phys. 70, 732 (1991).

[9] D. S. Sivia, W. A. Hamilton, and G. S. Smith, Physica B 173, 121 (1991)

[10] D. L. Worcester, Physica B 183, 139 (1991).

[11] J. S. Pedersen, J. Appl. Crystallogr. 25, 129 (1992).

[12] J. S. Pedersen and I. W. Hamley, J. Appl. Crystallogr. 27, 36 (1994).

[13] I. W. Hamley and J. S. Pedersen, J. Appl. Crystallogr. 27, 29 (1994).

[14] X.-L. Zhou and S.-H. Chen, Phys. Rev. E 47, 3174 (1993).

[15] X.-L. Zhou, L.-T. Lee, S.-H. Chen, and R. Strey, Phys.
Rev. A 46, 6479 (1992).

[16] N. Berk (unpublished).

[17] K. Kunz, J. Reiter, A. Götzelmann, and M. Stamm, Macromolecules 26, 4316 (1993).

[18] N. Singh, M. Tirrell, and F. Bates, J. Appl. Crystallogr. 26, 650 (1993).

[19] P. S. Pershan and J. Als-Nielsen, Phys. Rev. Lett. 52, 759 (1984).

[20] D. K. Schwartz, M. L. Schlossman, E. H. Kawamoto, G. J. Kellogg, P. S. Pershan, and B. M. Ocko, Phys. Rev. A 41, 5687 (1990).

[21] A. Braslau, M. Deutsch, P. S. Pershan, A. H. Weiss, J. Als-Nielsen, and J. Bohr, Phys. Rev. Lett. 54, 114 (1985).

[22] For example, see the following and the references therein: L. D. Chapman, D. R. Yoder, and R. Colella, Phys. Rev. Lett. 46, 1578 (1981).

[23] L. G. Parratt, Phys. Rev. 95, 359 (1954).

[24] L. B. Lurio, T. A. Rabedeau, P. S. Pershan, I. F. Silvera, M. Deutsch, S. D. Kosowsky, and B. M. Ocko, Phys. Rev. B 48, 9644 (1993).

[25] L. B. Lurio, T. A. Rabedeau, P. S. Pershan, I. F. Silvera, M. Deutsch, S. D. Kosowsky, and B. M. Ocko, Phys. Rev. Lett. 68, 2628 (1992).

[26] M. K. Sanyal et al., Europhys. Lett. 21, 691 (1993).

[27] P. S. Pershan, J. Phys. Condens. Matter 6, A37 (1994), and unpublished. 


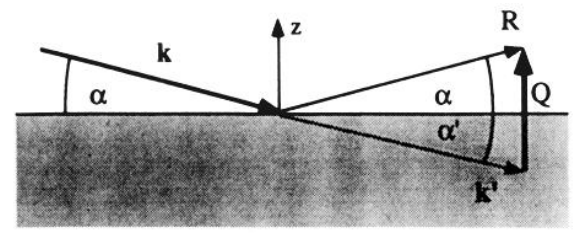

FIG. 1. Kinematics of the reflectivity geometry discussed in the text. 